\title{
Mutual interactions of oscillating quartz tuning forks in superfluid ${ }^{4} \mathrm{He}$
}

\author{
G. Sheshin and I. Gritsenko \\ B. Verkin Institute for Low Temperature Physics and Engineering of the National Academy of Sciences of Ukraine \\ 47 Lenin Ave., Kharkov 61103, Ukraine \\ E-mail: sheshin@ilt.kharkov.ua \\ D. Schmoranzer and L. Skrbek \\ Faculty of Mathematics and Physics, Charles University, V. Holešovičkách 2, Prague 8 18000, Czech Republic
}

Received March 21, 2013

\begin{abstract}
The quartz tuning fork has recently become a popular experimental tool for investigations of both classical and quantum turbulence in cryogenic helium. Its increased use in low-temperature experiments and a number of puzzling results obtained in the past have led to many questions concerning the interaction of multiple tuning forks or the interaction of tuning forks with other oscillators. We report measurements performed in He II at low temperatures around $360 \mathrm{mK}$, on the mutual interaction of tuning forks placed in the same volume of fluid, and examine the responsible mechanisms in an effort to discriminate between acoustic coupling and interaction via quantized vortices. To this end, the interaction of two tuning forks is investigated by analyzing their recorded resonance curves, looking for any nonelectrical crosstalk. Further, the force-velocity characteristics of a detector tuning fork are measured for different operating velocities of a generator tuning fork. As a complementary measurement, the intensity of sound waves is recorded using a set of miniature receivers. We confirm the current knowledge on acoustic emission by tuning forks in He II and verify properties of their radiation patterns. We conclude that in our experiment the interaction is almost entirely mediated by sound waves.
\end{abstract}

PACS: $67.25 . d g$ Transport, hydrodynamics, and superflow;

67.25.dt Sound and excitations.

Keywords: superfluid helium, interacting oscillators, acoustic emission.

\section{Introduction}

Since the discovery of superfluidity of ${ }^{4} \mathrm{He}$ by Kapitza [1], Allan and Misener [2] in 1938, experiments on various oscillatory systems in He II yielded many contributions to the current understanding of this interesting quantum fluid, not least of which was the pioneering work of Andronikashvili [3]. Today, many oscillating systems are still used to study various aspects of superfluidity and quantum turbulence in both isotopes of helium, ranging from oscillating wires, grids, spheres to a relatively more recent addition - quartz tuning forks.

The tuning forks are well-tested detectors of both classical and quantum turbulence in all the helium fluids cold He gas, normal liquid ${ }^{3} \mathrm{He}$ and ${ }^{4} \mathrm{He}$, their superfluid phases and even mixtures. They were also successfully employed for studies of cavitation in normal and superfluid helium [4], for measurements of viscosity [5,6], and more recently, their mutual interactions as observed in helium became of importance [7-9], and are seen, as we shall show, related to their capability to emit and absorb sound waves [10-12]. Depending on the parameters of the tuning fork, sound emission may even represent the dominant process of energy dissipation.

To study the mutual interaction of the tuning forks, we have constructed a dedicated cell optimized to allow measuring not only the influence of one fork on another, but also to measure the intensity of the sound waves emitted by the oscillating tuning forks and propagating in the helium liquid.

\section{Experimental setup}

The measurements were carried out in three experimental runs using a dilution refrigerator that allows reach- 
ing temperatures as low as $12 \mathrm{mK}$. The copper experimental cell was fitted with a silver heat exchanger and thermally connected to the mixing chamber. Either one or two tuning forks were placed in the cell in each experimental run (denoted F1, F2 in the first two runs, F3 and F4 together in the last run), in such a way that the ends of their prongs were located on the axis of symmetry of the cylindrical cell. Schematic arrangement of forks in the cell in all runs is shown in Figs. 1 (a)-(c). The experiments in He II were performed at temperatures $(360 \pm 10) \mathrm{mK}$ (forks $\mathrm{F} 1$ and F2), and (352 \pm 2 ) mK (forks F3 and F4). The parameters of the tuning forks are summarized in Table 1 . The cell also contains two sound receivers S1, S2 positioned across the cylindrical cell. These are piezoelectric sound sensors (type of material: PSI-5A-S4-ENH) with a resonant frequency of 4.7 MHz, and allow measurement up to $10 \mathrm{MHz}$. They were used to measure the intensity of sound waves in helium, as in our case the tuning forks can be expected to produce measurable acoustic signals [11,12].

The tuning forks were driven by ac voltage from two waveform generators and their signals were detected by two EG\&G Lock-in Analyzers 5208 using a common reference. The electronics used for the readout of the tuning forks are depicted in Fig. 1 (d). The voltage induced in one of the sound receivers was also optionally read by one of the lock-in amplifiers.
Table 1. Resonant frequencies, dimensions of the tuning forks used and their calibration constants. $L$ is the length of the prongs, $W$ stands for their width in the direction perpendicular to oscillation, $T$ denotes their thickness and $D$ is the separation between the prongs, both in the direction of oscillation. The dimensions are given as measured by optical microscopy. The tuning fork calibration constant, $a$, relates its mechanical properties to its electrical characteristics [5] and was determined using the selfcalibration method (measurement in vacuum).

\begin{tabular}{c|c|c|c|c|c|c}
\hline \hline Tuning fork & $\begin{array}{c}f_{\text {vac }}, \\
\mathrm{Hz}\end{array}$ & $\begin{array}{c}L, \\
\mathrm{~mm}\end{array}$ & $\begin{array}{c}W, \\
\mathrm{~mm}\end{array}$ & $\begin{array}{c}T, \\
\mathrm{~mm}\end{array}$ & $\begin{array}{c}D, \\
\mathrm{~mm}\end{array}$ & $\begin{array}{c}a \cdot 10^{6}, \\
\mathrm{C} \cdot \mathrm{m}^{-1}\end{array}$ \\
\hline \hline $\mathrm{F} 1$ & 32710.7 & 3.79 & 0.30 & 0.60 & 0.31 & 17 \\
$\mathrm{~F} 2$ & 32704.7 & 3.14 & 0.34 & 0.38 & 0.20 & 3.5 \\
F3 & 32720.3 & 3.14 & 0.34 & 0.38 & 0.20 & 2.69 \\
F4 & 32711.7 & 3.81 & 0.34 & 0.60 & 0.36 & 4.5 \\
\hline \hline
\end{tabular}

Based on the cell dimensions and the typical sound velocities of He II around $240 \mathrm{~m} / \mathrm{s}$ (in fact, in the experiments it would be close to $238 \mathrm{~m} / \mathrm{s}$ ), the fundamental longitudinal and transverse acoustic modes of the cell can be expected at frequencies around $5,5 \mathrm{kHz}$ and $15 \mathrm{kHz}$, respectively. The fundamental frequency corresponding to the resonance between the sound receivers ( $\approx 10 \mathrm{~mm}$ apart) is $12 \mathrm{kHz}$. Neglecting the complicated geometry of the tuning forks for purposes of the following estimation, the relevant har-
$22 \mathrm{~mm}$

(a)

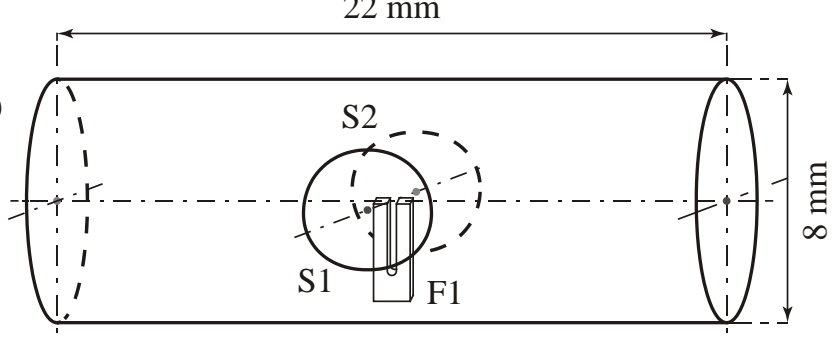

(b)

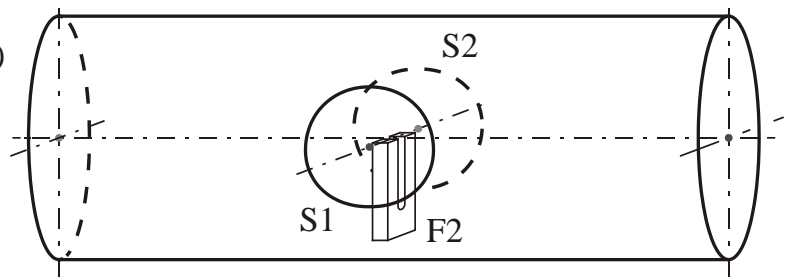

(c)

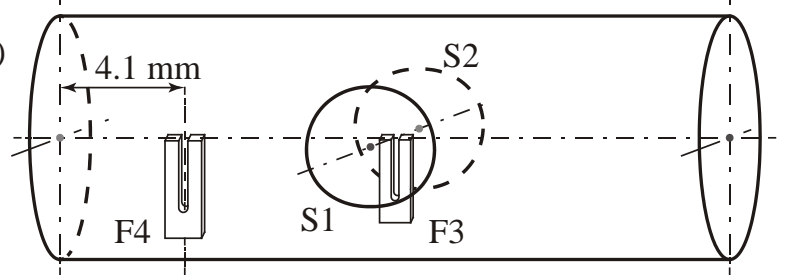

(d)

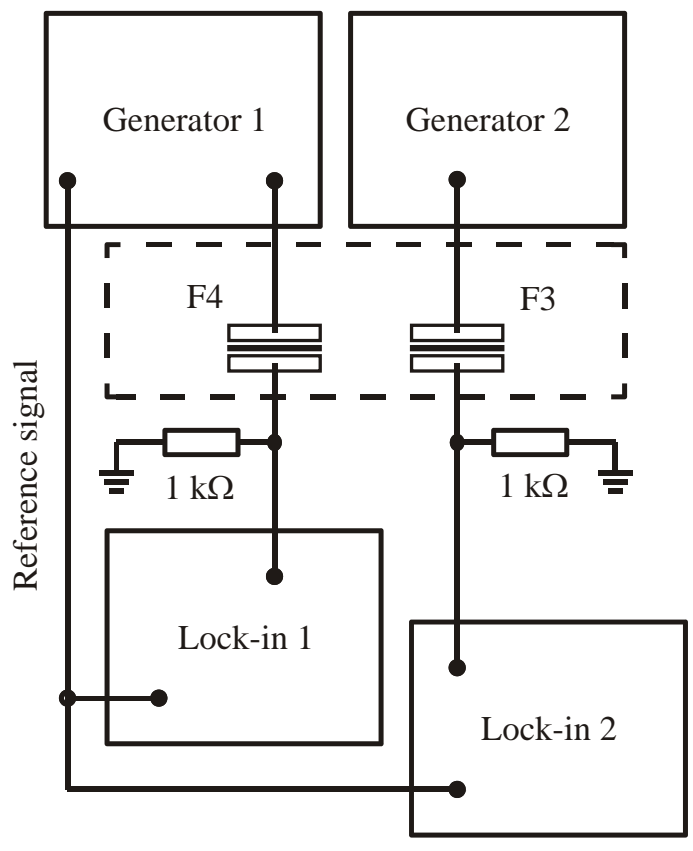

Fig. 1. Schematic depiction of the cylindrical cell of diameter $8 \mathrm{~mm}$ and height $22 \mathrm{~mm}$ thermally connected to the mixing chamber of a dilution refrigerator. The cell contains two sound receivers S1 and S2 separated by $\approx 10 \mathrm{~mm}$ and different tuning forks (F1, F2; F3, F4) placed in different positions in three subsequent experimental runs as shown. Note that F1 and F2 have different orientations with respect to the sound receivers S1, S2 (a)-(c). Schematics of the readout electronics shown for the case (c) in the left panel. Both lock-in amplifiers use the same reference signal in order to measure the influence of one tuning fork on another. The dashed line represents the boundary of the cryogenic parts of the setup (d). 
monics close to $32 \mathrm{kHz}$ that might potentially be excited by the tuning forks are therefore the first transverse harmonic around $30 \mathrm{kHz}$, and the fifth longitudinal harmonic expected close to $33 \mathrm{kHz}$. Conversely, the wavelength is $\approx 7.5 \mathrm{~mm}$ at $32.0 \mathrm{kHz}$ (estimate of tuning fork resonance frequency when immersed in superfluid He II).

While we cannot rule out the excitation of acoustic resonances of the cell altogether, it seems that they are rather sparsely spaced in the frequency domain (even with some reserve for smaller satellite peaks near the main resonances due to the complex 3D geometry) and, given that they are usually quite sharp in superfluid helium at low temperature $[8,11]$ and that the resonances of the tuning forks are extremely sharp themselves, we should be able to keep away from the acoustic standing modes during our measurements.

\section{Results and discussion}

First, the tuning forks were characterized at low temperature in vacuum and their calibration constants [5] were determined. Together with their measured resonant frequencies and their dimensions (denoted as in Ref. 5), they are listed in Table 1. The mutual interaction of F3 and F4 was also tested in vacuum to eliminate possibilities of purely electrical crosstalk or interaction via vibrations propagating through the solid walls of the cell. This was done in such a way that fork F3 was driven near its resonance and the signal of F4 was analysed both at the same frequency F3 was oscillating at, and at its own resonance frequency only $\approx 10 \mathrm{~Hz}$ below that of F3. Let us stress that in vacuum no measurable crosstalk was detected between the tuning forks - if it was present, it was definitely well below noise levels, meaning that any crosstalk measured in helium is indeed due to the presence of the fluid.

A similar test was also carried out for the signal from sound receivers, and here we found that oscillations of forks F3 and F4 resulted in comparable signals from the receiver S1 in vacuum and in helium, and hence that this signal is likely due to inductive or capacitive coupling. In the experiments with the tuning forks F1 and F2, the situation was much better and the sound receiver signals from these experiments will be discussed in due course.

After the vacuum measurements were completed in the arrangement with forks F3 and F4, He II was admitted into the cell, and at the temperature of $356 \mathrm{mK}$ and pressure 0.16 bar the resonance curves of F3 and F4 were recorded, giving resonant frequencies $31687 \mathrm{~Hz}$ (linewidth $10 \mathrm{~Hz}$ ) and $32005 \mathrm{~Hz}$ (linewidth $23 \mathrm{~Hz}$ ), respectively. It is important that there is virtually no overlap between the resonances and that neither distortions of the resonance curves nor extra peaks due to coupling to acoustic resonances of the cell were observed.

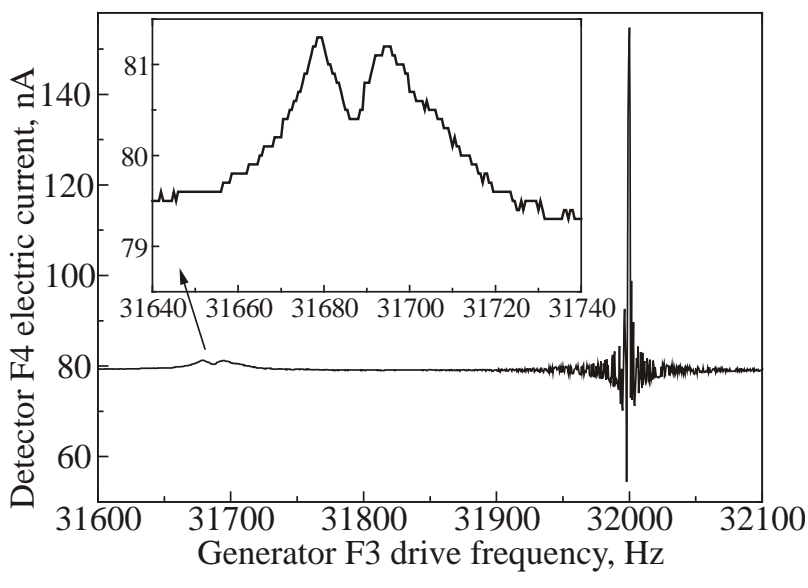

Fig. 2. The signal of the detector tuning fork F4 as measured by the lock-in amplifier referenced to the frequency of the drive of the generator tuning fork F3. A pair of smaller peaks is detected at the resonance of the generator fork. Oscillations of the generator fork F3 are partly transmitted via sound waves to the detector fork F4. A series of beats is also measured near the resonance frequency of F4, resulting from the small frequency differences between the driving voltage of F4 (in resonance) and the reference signal from the driving waveform generator connected to F3.

In the almost pure superfluid near $350 \mathrm{mK}^{*}$, there are two main ways in which different tuning forks could influence each other's behavior. One type of interaction can be mediated by first sound waves and another by quantized vortices. While second sound was found to be potentially important $[9,13,14]$ in the temperature range above $1 \mathrm{~K}$, its role is greatly diminished at these low temperatures. To discriminate between the two mechanisms proposed, we have performed several types of measurements.

In one set of measurements, we have been driving the fork F3 ("generator") near its resonance and monitoring the signal of the fork F4 ("detector") at the same frequency (using the common reference of the lock-in amplifiers) while driving it at its own resonance with a low voltage. This measurement should mainly be sensitive to acoustic waves propagating through the superfluid. The results are summarized in Fig. 2.

From the data, it is evident that the tuning forks do interact with each other, as a small but measurable signal near the resonance of F3 was read from F4 as well, clearly indicating a transfer of oscillatory motion at a specific frequency from the generator fork to the detector fork. Further, when the drive of F3 is shifted in frequency towards the resonance of F4, the signal of F4 - instead of reproducing a smooth resonance curve - starts to show beats resulting from the fine mismatch between the (low) drive

* The experiments were carried out at this relatively high temperature, because we use natural helium, containing also trace amounts of the isotope ${ }^{3} \mathrm{He}$. Its presence might have influenced results at temperatures below about $300 \mathrm{mK}$, as it constitutes an impurity and contributes to the drag forces. 
of F4 and the reference signal from the drive of F3, which are connected to the same lock-in amplifier.

Another type of measurement, more sensitive to the presence of quantized vortices (an analogical experiment with vibrating wires is described in Refs. 15, 16), relied on probing the transition to turbulence with each tuning fork, while the other was operated at its resonance with a fixed velocity amplitude. Among other, the results obtained with forks F3 and F4 are presented in Fig. 3 in terms of the force-velocity dependence and as drag coefficients. In our case, it is seen that while the linear drag may be somewhat shifted by operating the second tuning fork, the critical velocity and the turbulent drag are largely unaffected.

Note in passing that the drag coefficient in the limit of large velocities behaves differently for different tuning forks. If, for the fork F2 the limiting value is about one as in classical flows or as observed in Ref. 18, for F3, although the transition from linear to nonlinear drag regime is also displayed clearly, the limiting value of the drag coefficient is considerably lower than one [7].

We would like to point out that the tuning forks F1 and F4 (data in Fig. 3) exhibit much larger linear drag than F2 or F3 and did not reliably detect the transition to turbulence in these experiments. F1 and F4 are notably larger in size than their counterparts, and in accord with Refs. 11, 12 we attribute this large increase in the linear drag, roughly by one order of magnitude, to significant losses of energy via emitted sound waves. This is supported by the fact that for a constant velocity amplitude, the acoustic emission power [11,12] scales with the fork dimensions as $L^{2} W^{2} T^{2}(T+D)^{2}$, giving a factor of 10 between the emission powers of F3 and F4 and a factor of 7 between F3 and F1 (F2 differs from F3 only slightly, they have the same nominal dimensions). This is in good agreement with the data shown in Fig. 3.

The inability of forks F1 and F4 (due to strong acoustic emission) to detect the nonlinear turbulent drag does not mean that they are unable to generate quantum turbulence at the examined range of velocities, merely that the turbulent drag is not yet sufficiently large to become dominant. In fact, based on other measurements with similar tuning forks [19-21], it is extremely unlikely that at velocities around 80 or $90 \mathrm{~mm} / \mathrm{s}$, quantum turbulence would not be generated (also compare with the critical velocity of F2 and F3 in Fig. 3 between 20 and 30 mm/s).

Therefore, based on the experiment performed with the forks F3 and F4, we can say with moderate confidence that while these forks interact via sound waves and weakly affect their respective linear drag forces (which include drag due to acoustic emission), no significant influence on the critical velocities for the transition to turbulence or the turbulent drag was detected.
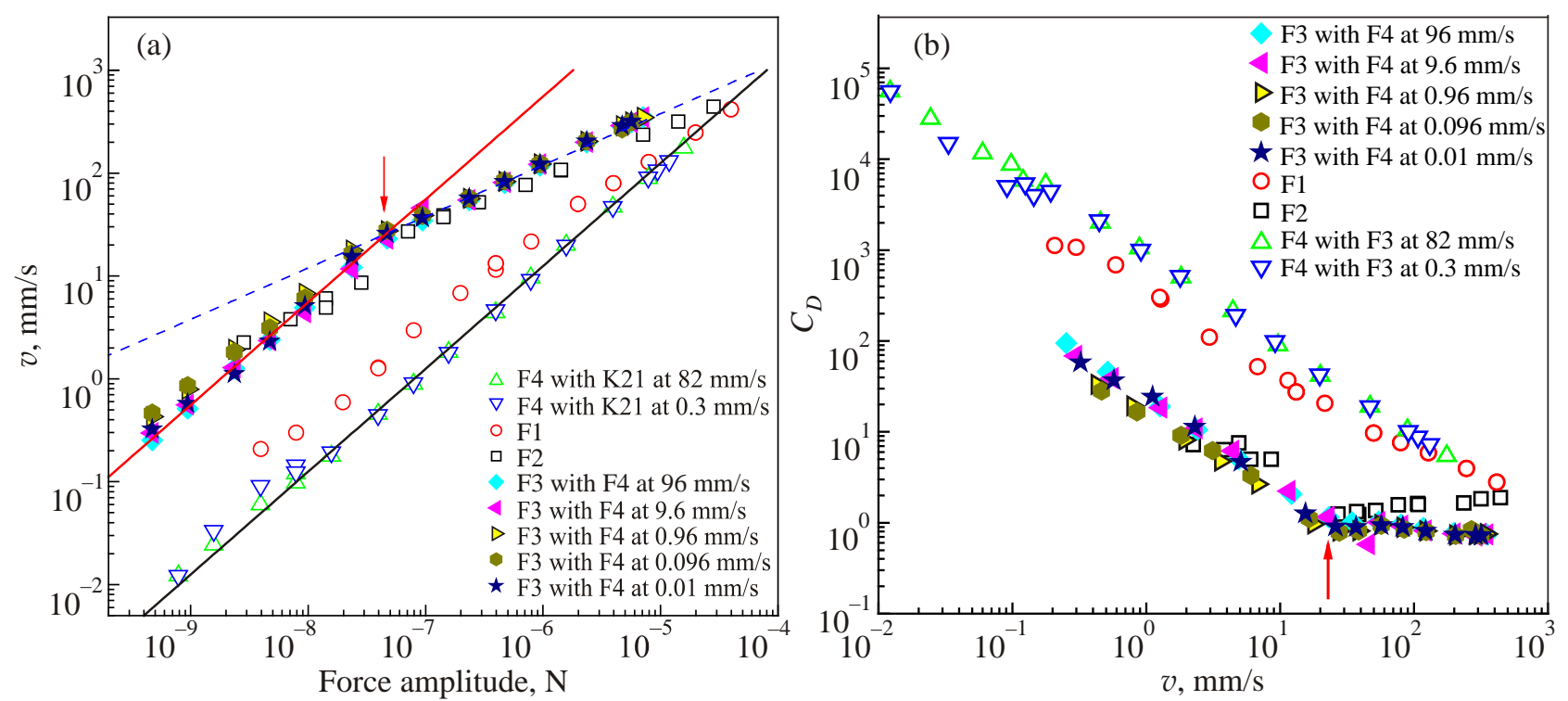

Fig. 3. (Color online) Velocity amplitude $v$ vs driving force $F$ (a) and the drag coefficient $C_{D}=2 F / \rho A v^{2}$ (b), where $A$ stands for the cross-sectional area of a prong of the tuning fork and $\rho$ for the fluid density. The forces and velocities were obtained using the calibration constants listed in Table 1 and the procedure detailed in Refs. 5, 17. The data are shown for all the tuning forks used in the three runs, and for the case of F3 and F4, their mutual interaction via quantized vortices was also studied by changing the fixed velocity amplitude of the other fork (as indicated) between successive measurements of the force-velocity curve. Note that no significant influence is observed on the magnitude of the drag force or the critical velocity. The forks F2 and F3 have very similar behavior, and both detect the transition to turbulence around 20 to $30 \mathrm{~mm} / \mathrm{s}$ (red arrow). F1 and F4 experience significantly larger drag at the same velocities (differing by at least one order of magnitude), which is related to energy losses due to acoustic emission (see text). Any drag force arising from (quantum-) turbulent flows around these two forks is screened by this acoustic drag and therefore no clear transition to turbulence is detected. The solid lines are linear dependences, the dashed line represents a quadratic law, characteristic of the turbulent drag regime. 

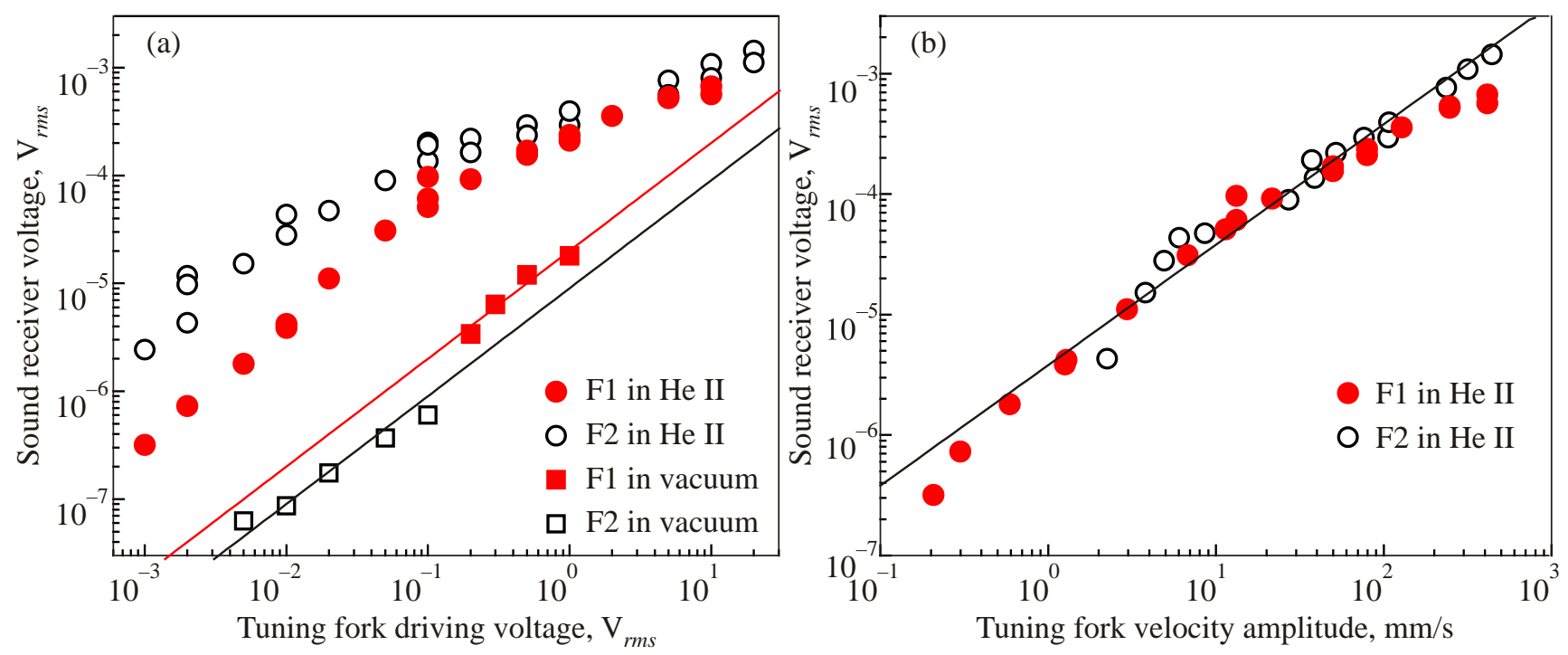

Fig. 4. (Color online) Voltage signal of the sound receiver S1 versus tuning fork driving voltage (a) and tip velocity amplitude (b). While electrical crosstalk in vacuum is observed for both tuning forks, it is significantly lower (20 times for F1 and 100 times for F2) than the signal measured in He II. This represents a direct observation of acoustic emission by the tuning forks, and confirms well to the fact that the measured signal seems to be directly proportional to the velocity amplitude and not the driving voltage. The solid lines are linear dependences.

Further we will discuss the signals from the sound receiver S1. As was mentioned earlier, for tuning forks F3 and F4, the signals of S1 in helium and in vacuum were comparable, and likely related to capacitive or inductive coupling. The results for tuning forks F1 and F2 are shown in Fig. 4, where receiver signal is plotted both versus the applied driving voltage and versus tuning fork (tip) velocity amplitude. In this case a clear difference between vacuum and helium is observed, implying that sound waves propagating through He II are indeed recorded, albeit together with a background due to electrical crosstalk. This statement is further supported by the fact that the signal voltage seems to be proportional to the velocity rather than the driving voltage.

Considering the relative magnitudes of acoustic emission by the forks F1 and F2 as discussed above (F1 roughly 7 times stronger), it may seem surprising that the signals from the receivers show almost equal levels at the same velocity amplitude. We attribute this to the different orientations of the tuning forks (see Fig. 1) - fork F2 oscillates in the direction of the axis joining the two sound receivers. This means that the spatial radiation pattern is rotated by 90 degrees with respect to that of the fork F1 and different intensities might be expected.

Upon consulting the radiation diagrams for tuning forks as shown in Fig. 4 of Ref. 10, we see that this could easily explain the apparent discrepancies in the recorded intensity of sound radiation, as higher sound levels are expected in the plane of oscillations than in the normal plane in distances $R=\lambda / 8$ as well as $R=3 \lambda / 4$, where $\lambda$ is the wavelength. In our case the receivers are in the distance $R \simeq 2 \lambda / 3$, i.e., in between the values taken from Ref. 10 . Thus, similar behavior can be expected, and if we substitute $R=2 \lambda / 3$ into Eq. (11) of Ref. 10, we indeed find that the ratio of the pressure amplitudes in the two principal directions is around 6.3, i.e., it almost exactly balances the ratio between the geometric factors in the emission powers of the two tuning forks ${ }^{*}$ and explains why similar sound levels for a given velocity amplitude are recorded by the receiver in both runs.

\section{Conclusions}

Our investigation of mutual interaction of quartz tuning forks in He II at low temperature confirms that such an interaction exists even in the linear drag regime and is mediated by sound waves emitted by either tuning fork. We also demonstrate the increase in the linear drag force due to this acoustic emission and its adverse effect on the capability of the tuning forks to detect quantum turbulence.

While in our experiments we have not observed any notable interaction via quantized vortices resulting in changes of the critical velocity, such an interaction cannot be ruled out, as several other experiments using different oscillating

* Note that the same geometric factor also appears in the expression for the pressure amplitude. If the velocity amplitude is used explicitly in the formula, the emission power will be expressed as linear in the pressure amplitude because the energy flux is given by $1 / 2 \operatorname{Re}\left(p v^{*}\right)$, where $p$ and $v$ stand for the oscillating parts of pressure and velocity, respectively, and the asterisk represents complex conjugation. See also Ref. 11. 
bodies have shown clear indications to the contrary $[15,16]$. The most likely reason why we have not seen this type of interaction is the large acoustic drag acting on our tuning forks, especially F1 and F4, effectively screening any lower drag forces that might arise from the generation of quantized vortices.

Finally and most importantly, we have for the first time observed the sound waves radiated by quartz tuning forks in cryogenic conditions directly and measured their amplitude by sensitive miniature sound receivers. While our experimental technique does not allow an exhaustive quantitative analysis of the radiation patterns, the obtained data are in reasonable agreement with the models presented in the literature.

\section{Acknowledgements}

This research is supported by the programs of bilateral cooperation between the National Academy of Sciences of Ukraine and the Academy of Sciences of the Czech Republic; by the fund of the National Academy of Sciences of Ukraine and the Russian Academy of Sciences 10-2012. DS and LS acknowledge the support of the programme UNCE 2040 of Charles University in Prague.

1. P. Kapitza, Nature 141, 74 (1938).

2. J.F. Allen and A.D. Misener, Nature 141, 75 (1938).

3. E.L. Andronikashvili, Zh. Eksp. Teor. Fiz. 18, 424 (1948).

4. M. Blažková, D. Schmoranzer, and L. Skrbek, Fiz. Nizk. Temp. 34, 380 (2008) [Low Temp. Phys. 34, 298 (2008)].

5. R. Blaauwgeers, M. Blažková, M. Človečko, V.B. Eltsov, R. de Graaf, J. Hosio, M. Krusius, D. Schmoranzer, W. Schoepe, L. Skrbek, P. Skyba, R.E. Solntsev, and D.E. Zmeev, J. Low Temp. Phys. 146, 537 (2007).

6. H. Divišová, J. Lang, M. Rotter, and D. Schmoranzer, in: Experimental Fluid Mechanics 2011, Intern. Conf. Exp. Fluid Mechanics, Jičin, Czech Republic, November 22-25 (2011).
7. D. Garg, V.B. Efimov, M. Giltrow, P.V.E. McClintock, L. Skrbek, and W.F. Vinen, Phys. Rev. B 85(14), 144518 (2012).

8. D. Garg, V.B. Efimov, M. Giltrow, P.V.E. McClintock, and L. Skrbek, Fiz. Nizk. Temp. 38, 1300 (2012) [Low Temp. Phys. 38, 1026 (2012)].

9. D. Schmoranzer, M. La Mantia, and L. Skrbek, J. Low Temp. Phys. 171, 226 (2013).

10. R.M. Sillitto, Am. J. Phys. 34, 639 (1966).

11. D. Schmoranzer, M. La Mantia, G. Sheshin, I. Gritsenko, A. Zadorozhko, M. Rotter, and L. Skrbek, J. Low Temp. Phys. 163, 317 (2011).

12. D.I. Bradley, M. Človečko, S.N. Fisher, D. Garg, E. Guise, R.P. Haley, O. Kolosov, G.R. Pickett, V. Tsepelin, D. Schmoranzer, and L. Skrbek, Phys. Rev. B 85, 014501 (2012).

13. E. Pentti, J. Tuoriniemi, A. Salmela, and A. Sebedash, J. Low Temp. Phys. 150, 555 (2008).

14. E. Pentti, J. Rysti, A. Salmela, A. Sebedash, and J. Tuoriniemi, J. Low Temp. Phys. 165, 132 (2011).

15. R. Goto, S. Fujiyama, H. Yano, Y. Nago, N. Hashimoto, K. Obara, O. Ishikawa, M. Tsubota, and T. Hata, Phys. Rev. Lett. 100, 045301 (2008).

16. H. Yano, T. Ogawa, A. Mori, Y. Miura, Y. Nago, K. Obara, O. Ishikawa, and T. Hata, J. Low Temp. Phys. 156, 132 (2009).

17. G.A. Sheshin, A.A. Zadorozhko, E.Y. Rudavskii, V.K. Chagovets, L. Skrbek, and M. Blazhkova, Fiz. Nizk. Temp. 34, 1111 (2008) [Low Temp. Phys. 34, 875 (2008)].

18. D.I. Bradley, M.I. Fear, S.N. Fisher, A.M. Guenault, R.P. Haley, C.R. Lawson, P.V.E. McClintock, G.R. Pickett, R. Schanen, V. Tsepelin, and L.A. Wheatland, J. Low Temp. Phys. 156, 116 (2009).

19. M. Blažková, D. Schmoranzer, and L. Skrbek, Phys. Rev. E 75(2), 025302 (2007).

20. M. Blažková, D. Schmoranzer, L. Skrbek, and W.F. Vinen, Phys. Rev. B 79(5), 054522 (2009).

21. D. Schmoranzer, M. Králová, V. Pilcová, W.F. Vinen, and L. Skrbek, Phys. Rev. E 81(6), 066316 (2010). 